\title{
A Mouse Model of Acute Q Fever Following Infection Via A Non-Invasive Intratracheal Inoculation Method
}

\section{Xueyuan $\mathrm{Hu}$}

State Key Laboratory of Pathogen and Biosecurity, Beijing Institute of Microbiology and Epidemiologu; College of Life Sciences, Hebei Normal University

\section{Yonghui Yu}

State Key Laboratory of Pathogen and Biosecurity, Beijing Institute of Microbiology and Epidemiology Junxia Feng

State Key Laboratory of Pathogen and Biosecurity, Beijing Institute of Microbiology and Epidemiology

Mengjiao Fu

State Key Laboratory of Pathogen and Biosecurity, Beijing Institute of Microbiology and Epidemiology

\section{Lupeng Dai}

State Key Laboratory of Pathogen and Biosecurity, Beijing Institute of Microbiology and Epidemiology

\section{Zhiyu Lu}

State Key Laboratory of Pathogen and Biosecurity, Beijing Institute of Microbiology and Epidemiology

\section{Zemin He}

State Key Laboratory of Pathogen and Biosecurity, Beijing Institute of Microbiology and Epidemiology

Wenbo Luo

State Key Laboratory of Pathogen and Biosecurity, Beijing Institute of Microbiology and Epidemiology

\section{Xiaolu Xiong}

State Key Laboratory of Pathogen and Biosecurity, Beijing Institute of Microbiology and Epidemiology Jinglin Wang

State Key Laboratory of Pathogen and Biosecurity, Beijing Institute of Microbiology and Epidemiology

Dongsheng Zhou

State Key Laboratory of Pathogen and Biosecurity, Beijing Institute of Microbiology and Epidemiology

\section{Bohai Wen}

State Key Laboratory of Pathogen and Biosecurity, Beijing Institute of Microbiology and Epidemiology

\section{Baohua Zhao}

Hebei Normal University College of Life Sciences

Jun Jiao ( $\square$ jiaojun51920@sina.com )

State Key Laboratory of Pathogen and Biosecurity, Beijing Institute of Microbiology and Epidemiology https://orcid.org/0000-0002-4041-1782 
Research article

Keywords: Q fever, Coxiella burnetii, mouse model, intratracheal inoculation

Posted Date: April 15th, 2019

DOI: https://doi.org/10.21203/rs.2.9192/v1

License: (c) (i) This work is licensed under a Creative Commons Attribution 4.0 International License. Read Full License 


\section{Abstract}

Background: Q fever is a worldwide zoonosis caused by Coxiella burnetii and mainly transmitted by aerosols. This study aims at establishing a systematic and efficient mouse model of acute $Q$ fever via intratracheal (IT) inoculation of aerosolized C. burnetii. Methods: BALB/c mice were infected with C. burnetii via IT route using a non-invasive aerosol pulmonary delivery device to directly place the living $\mathrm{C}$. burnetii organisms into their tracheas. The bacterial loads, pathological lesions, and serological responses were analyzed in mice, and compared with those of mice infected via intraperitoneal (IP) route. Results: As early as at day three post-infection (pi) with a low dose of $\mathrm{C}$. burnetii ( $1 \times 10^{4}$ per mouse), a large amount of $\mathrm{C}$. burnetii organisms were determined in blood, lungs, hearts, livers, and spleens of the mice. The inflammatory infiltration was observed in hearts and lungs of mice. Compared with mice infected via IP route, the mice infected via IT route exhibited a higher level of bacterial loads and more severe pathological lesions in hearts and lungs at day 3 and day 7 pi. Conclusions: These data indicated that IT route is more efficient than IP route to cause acute $\mathrm{C}$. burnetii infection in mice. Overall, we successfully established a mouse model of $\mathrm{C}$. burnetii infection via IT route, which is useful for investigations of pathogenesis and immunity of acute $C$. burnetii infection as well as evaluation of therapeutic drugs and preventive vaccines of $Q$ fever.

\section{Background}

Q fever is a worldwide zoonosis caused by $C$. burnetii, an intracellular Gram-negative bacterium. $C$. burnetii infection exhibits various acute and chronic clinical manifestations in human. Acute $Q$ fever is generally a flu-like illness with high fever, headache, malaise, and myalgia [1], while chronic Q fever frequently presents endocarditis [2] and/or hepatitis [3] and occasionally appears osteomyelitis [4].

C. burnetii is able to infect a wide range of animals and livestock, sheep, goats, and cattle are its major reservoirs $[5,6]$. Most human acute $C$. burnetii infections occur after direct exposure to infected animals and their products (placenta, abortion products, hides, wool, manure, etc.). The occupational exposures of persons such as farmers, veterinarian, and abattoir workers in close contact with these livestock have a higher risk of $Q$ fever [7-9]. Q fever has been recognized as an important infectious disease in many countries including China, and a very large outbreak of Q fever 2007 to 2010 in the Netherlands has shown that $\mathrm{Q}$ fever could become a major public problem [10].

Animal models of acute $C$. burnetii infection are usually prepared with mice, guinea pigs, or nonhuman primates [11], and mice are the most attractive animal models such as BALB/c and SCID mice and can be analyzed easily by various molecular, cellular, and immunological techniques and methods [12]. The aerosolization most closely resembles the route of natural $C$. burnetii infection in human, acute $C$. burnetii infection of mice may be caused by $C$. burnetii aerosolization using the whole-body aerosol exposure apparatus, but it depends on particle size and the anatomical parameters of the animal and the procedure requires a higher titer of organisms to achieve study dosages within the lungs [13, 14]. In addition, the release of $C$. burnetii aerosol by the aerosol exposure apparatus may cause abrupt infection 
of animals, and it remains uncertain if the systemic dissemination of the infection only comes from the lungs of animals or also this involves extensive and complicated laboratory procedures [5, 15-17].

The other way to cause acute $C$. burnetii infection of mice in laboratories is to inoculate the organism directly into mice. Generally, there are three routes for the direct inoculation of $C$. burnetii: IP, IT and intranasal (IN) route [5, 18-21]. The IP inoculation is a convenient route, by which organisms quickly enter the circulation for systemic dissemination, involving many organs such as heart, lung, lymph nodes, and bone marrow of the mice [21,22], but it does not resemble the route of natural infection of $C$. burnetii in human. IN inoculation of $C$. burnetii closely resembles the route of natural infection in human because the organisms may rapidly enter the lungs of the mice and cause pneumonia. However, by IN inoculation, only the minority of $C$. burnetii inoculum are applied the nares and respired through the nasal passages into the lungs of the mice and the majority remains within the nasal passages [22, 23].

Unlike IN inoculation, IT inoculation provides more closely resemble the route of natural $C$. burnetii infection in human, which directly place a known quantity of organisms into the tracheas by passing the nasal passages of the mice [24]. In the present study, a mouse model of acute $C$. burnetii infection was developed with the immunocompetent mice (BALB/C) via IT inoculation of $C$. burnetii using a noninvasive aerosol pulmonary delivery device. The bacterial loads, pathological lesions, and serological responses were analyzed in mice infected with different doses of living $C$. burnetii organisms.

\section{Methods}

\section{Bacteria}

C. burnetii Xinqiao strain (phase I virulent strain) was cultured in Acidified Citrate Cysteine Medium (ACCM) passaged for 2 times in a $2.5 \%$ oxygen environment and purified by high speed centrifugation as previously described [25]. The purified organisms were suspended in phosphate-buffered saline buffer (PBS) and stored at $-70^{\circ} \mathrm{C}$.

\section{Animals}

SPF (specific pathogen free) BALB/c mice (Female, 6-8 weeks old) purchased from Vital River Laboratories (Beijing, China) were housed in sterile microisolator cages under a specific pathogen-free condition in the animal biosafety level-3 (ABSL-3) laboratory.

\section{Infection}

All animal care and procedures were in accordance with institutional policies for animal health and wellbeing and approved by the Institute of Animal Care and Use Committee (IACUC) of Academy of Military Medical Science (AMMS). Mice were randomly divided into 3 groups (9 mice per group) and they were infected with $1 \times 10^{4}$ (low dose), $1 \times 10^{6}$ (medium dose), and $1 \times 10^{8}$ (high dose) C. burnetii organisms suspended in $50 \mu \mathrm{l}$ PBS via IT route, respectively. The suspension of $C$. burnetii was inoculated directly 
into the tracheas of BALB/c mice using the MicroSprayer aerosolizer (Huironghe Company, Beijing, China) as previously described [26]. Briefly, mice were anaesthetized by intraperitoneal injection of pentobarbital sodium (100 mg/kg body weight), and then each mouse was placed on the slanted board ( $60^{\circ}$ angle from the horizontal direction) and supported by a nylon band under its upper incisors, and footpad reflex was checked to confirm it was asleep. After a clear view of the trachea under laryngoscope (Huironghe Company, Beijing, China), the mouth cavity was lightened with shades of orange-red and the trachea appeared as a white light spot, and then the MicroSprayer aerosolizer was inserted $25 \mathrm{~mm}$ from the larynx (near the tracheal bifurcation) and $50 \mu$ of suspension was sprayed. In addition, 9 mice were intraperitoneally infected with $1 \times 10^{8} \mathrm{C}$. burnetii organisms as control.

At day 3,7 , and 14 post-infection (pi), 3 mice in each group were euthanized through cervical dislocation, respectively, and their blood samples were collected to separate sera, their bodies and spleens were weighted, and their hearts, livers, spleens, and lungs were aseptically collected individually. Each organ was divided into two parts, one for extraction of total genomic DNA used in the real-time quantitative polymerase chain reaction (qPCR) and the other for histopathological analysis. Animal carcasses were safely disposed by the IACUC.

\section{qPCR specific for $C$. burnetii}

Ten mg of each organ sample and $100 \mu \mathrm{l}$ of blood per mouse were used to extract total genomic DNA with a DNeasy Blood \&Tissue kit (Qiagen, $\mathrm{GmbH}$, Germany) as described by the manufacturer. Each DNA sample was eluted from the DNA extraction column with $200 \mu$ l elution buffer. Two $\mu$ l per DNA sample was used for each reaction in qPCR with primers dotAF (5'- CCATGGCCCCAATTCTCTT -3') and dotAR (5'GCGCAATACGCTCAATCACA -3') and a probe (5'6-FAM CCGGAGATACCGGCGGTGGG 3'TAMRA-N) targeting the $\operatorname{dot} A$ gene of $C$. burnetii.

\section{Histopathological analysis}

Each organ sample was fixed in $10 \%$ buffered formalin for a minimum of $48 \mathrm{~h}$, and then the sample was sectioned and embedded in paraffin. The paraffin- embedded tissue was sliced into a small thickness allowed to adhere to slides, and the slides were stained with hematoxylin-eosin (HE) to observe pathological changes under light microscopy.

\section{Detection of IgGs to $C$. burnetii by ELISA}

By enzyme-linked immunosorbent assay (ELISA), IgGs to phase I or phase II antigen of $C$. burnetii were determined in each mouse serum in 96-well polystyrene plates (NUNC, Shanghai, China) coated with 100 $\mu \mathrm{l}$ of $1 \times 10^{8}$ formalin-killed C. burnetii Xinqiao strain whole cells (phase I antigen) and Grita strain whole cells (phase II antigen), respectively, according to described previously [27].

\section{Statistical analysis}


The results of qPCR, the ratios of spleen to body weight and the thickness of diaphragmatic walls of the mice were expressed as the mean \pm SD of 3 mice per group. The differences in $C$. burnetii loads, in $O D_{450}$ of antibody response in sera, in the thickness of diaphragmatic walls in the lungs and in the ratios of spleen to body weight were assayed using the two-way analysis of variance (ANOVA) test followed by least significant difference (LSD) test and Student-Newman-Keuls (SNK) test in Statistical Package in the Social Sciences ver. 19 software (SPSS, Chicago, IL). $P<0.05$ was considered significantly different.

\section{Results}

\section{Clinical symptom of mice}

To compare the clinical symptom of $C$. burnetii infection by different dose and different route, we infected mice with $1 \times 10^{4}, 1 \times 10^{6}$, or $1 \times 10^{8}$ of $C$. burnetii via IT route and $1 \times 10^{8}$ of $C$. burnetii via IP route and monitored the development of symptoms and survival of the mice. Approximate three days after infection with $C$. burnetii, all mice showed the signs of illness, including ruffled coats and decreased activity, but none of the mice died. The mice infected via IT route exhibited more severe clinical signs of $Q$ fever than the mice infected via IP route.

\section{The $C$. burnetii distribution in mice}

To compare the bacterial dissemination in mice infected with different dose $\left(1 \times 10^{4}, 1 \times 10^{6}, 1 \times 10^{8}\right)$ of $C$. burnetii via IT route, 3 mice per group were killed at day 3, 7, or $14 \mathrm{pi}$, and the bacterial loads in their blood, hearts, lungs, livers and spleens were detected by qPCR, and their bodies and spleens were weighted. As shown in Fig. 1, a large amount of $C$. burnetii were detected in these organs of all the mice infected via IT route at day 3 pi. The bacterial loads in 4 organs at day 7 pi were higher than those at day 3 or 14 pi, respectively. There were not significantly increased or decreased in bacterial loads in hearts, lungs, livers, or spleens of the mice infected via IT route with each dose of $C$. burnetii between three time points pi. A large amount of $C$. burnetii were also detected in blood, but the results calculated were not normally distributed (data not shown).

Next, we compared the bacterial dissemination in mice infected with high dose $\left(1 \times 10^{8} /\right.$ per mouse) of $C$. burnetii via IT route and via IP route. Interestingly, the bacterial loads in hearts, but not in other organs, of mice infected via IT route were significantly higher than those of the mice infected via IP route at day 3 and $7 \mathrm{pi}$, while the bacterial loads in lungs of mice infected via IT route were modestly higher than those of mice infected via IP route at each time point pi, but not significantly different (Fig. 2a, b, c, d). In addition, the ratio of spleen to body weight of mice infected via IT route at day 3 and 14 pi were significantly higher than those of mice infected via IP route (Fig. 2e).

\section{Histopathological findings}

To evaluate the histopathological lesions caused by $C$. burnetii infection via IT route, we examined the stained tissue slices from heart, lung, spleen, and liver of mice using a light microscopy. The infiltration of 
inflammatory cells was found in epicardium (Fig. 3a) and myocardium (Fig. 3b) of all mice infected via IT route at day $3 \mathrm{pi}$, and the inflammatory cells in hearts increased gradually at day 7 and $14 \mathrm{pi}$. There was no significant difference in numbers of inflammatory cells between mice infected with different dose of C. burnetii via IT route (data not shown). In contrast, only a small number of inflammatory cells were found in epicardium (Fig. 3a) and myocardium (Fig. 3b) of mice infected via IP route at day14 pi.

The infiltration of inflammatory cells, mainly neutrophils and macrophages, and the increased thickness of diaphragmatic walls were observed in lungs of mice infected with C. burnetii via IT route at day 3,7 , and $14 \mathrm{pi}$, while these histopathological lesions in lung of mice infected via IP route cannot be observed until day 7 pi (Fig. 4a). Inflammation cells and epithelial cells appeared in tracheas of mice at day 14 pi (Fig. 4b). There was no significant difference in numbers of inflammatory cells and thickness of diaphragmatic walls in lungs between mice infected with different dose of $C$. burnetii via IT route (data not shown). However, the thickness of diaphragmatic walls in lungs of mice infected with high dose of $C$. burnetii by IT route was significantly higher than that of mice infected with high dose of $C$. burnetii by IP route (Fig. 2f).

The pathological lesions were also observed in livers and spleens of mice infected with $C$. burnetii by IT route at day $7 \mathrm{pi}$. The infiltration of inflammation cells, mainly mononuclear cells, was focused on the portal area in livers (Fig. 5). Red pulp widened and white pulp atrophied, and splenic sinusoid extended with hyperemia, and the boundary became blurred in spleens (Fig. 6). The pathological lesions in livers and spleens were much severer at day $14 \mathrm{pi}$, specifically with high dose of $C$. burnetii. The pathological lesions in livers and spleens of mice infected with $C$. burnetii via IP route were observed at day 7 pi but they were lighter than those in mice infected via IT route.

\section{Serologic response to infection of $C$. burnetii}

To better understand the humoral immune response of mice against $C$. burnetii infection via IT route, we determined the antibody levels of IgGs to phase I antigen and to phase II antigen by ELSIA. As shown in Fig. 7, the antibody levels to phase I antigen (Fig. 7a) and to phase II antigen (Fig. 7b) in sera from mice infected with $C$. burnetii via both IT and IP route increased gradually throughout the infection. The antibody levels to phase II antigen were higher than those to phase I antigen, indicating an acute stage of C. burnetii infection. At the same time point, the antibody levels of mice infected via IT route were not significantly different from those of mice infected via IP route.

\section{Discussion}

The animal models of acute $C$. burnetii infection, simulating the natural route of infection and clinical presentations associated with human acute $\mathrm{Q}$ fever, had been developed with guinea pigs, mice, or nonhuman primates, and mice are the most attractive for being easy to perform for various research purposes in laboratories $[17,28,29]$. Previous studies showed that the aerosol inhalation procedure of infection in mice is of relevance to the majority of the acute $Q$ fever cases in human, and the clinical signs, bacterial burdens, and pathological alterations in certain mouse models may be used to monitor 
progression of the disease. The whole-body aerosol inhalation procedure was used to prepare the mouse models of the acute $C$. burnetii infection $[12,17,30]$, but it may cause systemic dissemination of the infection in mice by other routes besides the respiratory tract and also its inoculum dose of $C$. burnetii is not standardized. In the present study, the mouse model of the acute $C$. burnetii infection was developed via IT inoculation to directly place the living $C$. burnetii into the tracheas of the immunocompetent $\mathrm{BALB} / \mathrm{C}$ mice using a non-invasive aerosol pulmonary delivery device.

In this study, high levels of bacterial loads were determined in all four organs at any time point pi, and the highest levels of bacterial loads in these organs were determined at day $7 \mathrm{pi}$, suggesting $C$. burnetii was rapidly disseminated from the lungs to the deep organs of the mice and demonstrating $C$. burnetii grew stably and proliferated rapidly. Similar tendency of bacterial loads were determined in spleens of BALB/c mice infected by $C$. burnetii aerosolization [12]. Compared to other organs of mice infected with $C$. burnetii via IT route, the bacterial load in spleens was the highest, indicating a larger amount of organisms moved into the spleens through the circulatory system and then were engulfed by phagocytic cells and proliferated in the host cells in spleens [31]. In addition, the bacterial loads of mice infected via IT route at day 14 pi was lower than that at day $7 \mathrm{pi}$, suggesting that the immune system of the mice was activated to resist the infection and the mice were recovered in the late phase of the infection.

The pneumonia is a common complication during the acute $C$. burnetii infection in human because the lung is the earliest target organ of $C$. burnetii in natural infection [32]. In the mice infected with $C$. burnetii via IT route, the lungs were the foci of infection and the pathological lesions in lungs were more pronounced than those in livers and spleens. Isolated hepatitis is a frequent presentation of the acute $C$. burnetii infection in human. Besides granulomatous hepatitis and typical "doughnut" granulomas, atypical pathological changes were reported, such as epithelioid granuloma extensive extravasated fibrin and acute cholangitis without granuloma [33]. The infiltration of inflammation cells in livers of mice infected with $C$. burnetii via IT route, suggesting that the severe pathological lesions in livers of mice.

The cases of pericarditis, acute myocarditis, and acute endocarditis were reported in acute $C$. burnetii infection in human [34]. A large amount of $C$. burnetii organisms were determined in hearts and many inflammatory cells in epicardium and the infiltration of inflammatory cells in myocardium were observed of mice infected with $C$. burnetii via IT route at day 3 pi, suggesting early epicarditis in mice and the pathological lesions in hearts of mice were compatible with the heart diseases appearing in acute $C$. burnetii infection in human. It is speculated that the $C$. burnetii organisms arrived at the bronchi directly and rapidly reached the hearts through the pulmonary aorta without extensive circulatory system in mice infected with $C$. burnetii via IT route. The pathological lesions were consistent with the levels of bacterial loads in the organs of the mice, proving that the infection of $C$. burnetii spread hematogenously from the lungs to other organs such as the livers and spleens.

The influence of the inoculum dose in pathological lesions during $C$. burnetii infection was previously studied in guinea pigs [21]. A dose of $1 \times 10^{8} \mathrm{C}$. burnetii organisms was required to cause pathological lesions at the early phase of infection in the organs of mice infected by $C$. burnetii aerosolization $[5,9]$, 
suggesting that a large amount of $C$. burnetii organism must be present in aerosols for the development of $Q$ fever. In the present study, mice were infected with a low dose of $C$. burnetii organisms $\left(1 \times 10^{4} \mathrm{per}\right.$ mouse) via IT route, and the pathological lesions were observed in hearts and lungs as early as at day 3 pi and in all four organs at day $7 \mathrm{pi}$, suggesting the IT route was more efficient to cause acute $C$. burnetii infection in mice compared to aerosolization.

The specific IgGs to phase I and II antigen of $C$. burnetii were detected in sera from mice at day 7 and 14 pi even with the low dose of $C$. burnetii infection via IT route. The levels of the specific IgGs of mice infected with $C$. burnetii via IT route were not significantly different from those of mice infected via IP route, suggesting that the infection with $C$. burnetii via IT route elicited specific immune responses against $C$. burnetii like that via IP route.

\section{Conclusions}

In conclusion, the results in the present study confirm that the BALB/c mouse model of acute $C$. burnetii infection was successfully established by IT inoculation with $C$. burnetii using a non-invasive aerosol pulmonary delivery device. $C$. burnetii was accurately delivered into the tracheas of the mice, which modeled the pulmonary route of acute $C$. burnetii infection to avoid potential confounding complications caused by other routes of infection and simulated organ dissemination of organisms as seen during acute $C$. burnetii infection in human. This mouse model is useful for investigations of pathogenesis and immunity of acute $C$. burnetii infection as well as evaluation of therapeutic drugs and preventive vaccines of Q fever.

\section{Abbreviations}

IT: Intratracheal; pi: Post-infection; IP: Intraperitoneal; IN: Intranasal; ACCM: Acidified Citrate Cysteine Medium; PBS: Phosphate-buffered saline buffer; ABSL-3: Animal biosafety level-3; qPCR: Real-time quantitative polymerase chain reaction; HE: Hematoxylin-eosin; ELISA: Enzyme-linked immunosorbent assay; ANOVA: Analysis of variance; LSD: Least significant difference; SNK: Student-Newman-Keuls; SPSS: Statistical Package in the Social Sciences.

\section{Declarations}

\section{Ethics approval and consent to participate}

The study has been approved by AMMS, and all animal care and procedures were in accordance with institutional policies for animal health and well-being and approved by the IACUC of AMMS.

\section{Availability of data and materials}

The corresponding author would like to provide the data that support the findings of this study upon reasonable request. 


\section{Competing interests}

The authors have no conflict of interest to declare.

\section{Funding}

This work was supported by SKLPBS1802 and the Beijing Nova Program (Z181100006218116). The funders had no role in study design, data collection and analysis, decision to publish, or preparation of the manuscript.

\section{Authors' contributions}

Conceived and designed the experiments: $\mathrm{BHZ}$ and JJ. Performed the experiments: $\mathrm{XYH}$ and YHY. Collected and analyzed the data: XYH, JJ, JXF, MJF, LPD, ZYL, ZMH and WBL. Reviewed and Edited the manuscript: JLW, XLX, DSZ and BHW. All authors read and approved the final manuscript.

\section{Acknowledgements}

Not applicable.

\section{Consent for publication}

Not applicable.

\section{References}

1. Maurin M, Raoult D. Q fever. Clin Microbiol Rev. 1999; 12(4):518-53.

2. Gami AS, Antonios VS, Thompson RL, Chaliki HP, Ammash NM. Q fever endocarditis in the United States. Mayo Clin Proc. 2004; 79(2):253-7.

3. Chang K, Yan JJ, Lee HC, Liu KH, Lee NY, Ko WC. Acute hepatitis with or without jaundice: a predominant presentation of acute Q fever in southern Taiwan. J Microbiol Immunol Infect. 2004; 37(2):103-8.

4. Nourse C, Allworth A, Jones A, Horvath R, McCormack J, Bartlett J, et al. Three cases of Q fever osteomyelitis in children and a review of the literature. Clin Infect Dis. 2004; 39(7):e61-6.

5. Stein A, Louveau C, Lepidi H, Ricci F, Baylac P, Davoust B, et al. Q fever pneumonia: virulence of Coxiella burnetii pathovars in a murine model of aerosol infection. Infect Immun. 2005; 73(4):246977.

6. Sellens E, Bosward KL, Willis S, Heller J, Cobbold R, Comeau JL, et al. Frequency of Adverse Events Following Q Fever Immunisation in Young Adults. Vaccines (Basel). 2018; 6(4).

7. Bernasconi MV, Casati S, Peter O, Piffaretti JC. Rhipicephalus ticks infected with Rickettsia and Coxiella in Southern Switzerland (Canton Ticino). Infect Genet Evol. 2002; 2(2):111-20.

8. Lang GH. Q fever. Vet Rec. 1988; 123(22):582-3. 
9. Stein A, Raoult D. Pigeon pneumonia in provence: a bird-borne Q fever outbreak. Clin Infect Dis. 1999; 29(3):617-20.

10. Roest HI, Tilburg JJ, van der Hoek W, Vellema P, van Zijderveld FG, Klaassen $\mathrm{CH}$, et al. The Q fever epidemic in The Netherlands: history, onset, response and reflection. Epidemiol Infect. 2011;139(1):112.

11. Russell-Lodrigue KE, Andoh M, Poels MW, Shive HR, Weeks BR, Zhang GQ, et al. Coxiella burnetii isolates cause genogroup-specific virulence in mouse and guinea pig models of acute $Q$ fever. Infect Immun. 2009; 77(12):5640-50.

12. Melenotte C, Lepidi H, Nappez C, Bechah Y, Audoly G, Terras J, et al. Mouse Model of Coxiella burnetii Aerosolization. Infect Immun. 2016; 84(7):2116-23.

13. Brain JD, Knudson DE, Sorokin SP, Davis MA. Pulmonary distribution of particles given by intratracheal instillation or by aerosol inhalation. Environ Res. 1976; 11(1):13-33.

14. Munder A, Krusch S, Tschernig T, Dorsch M, Luhrmann A, van Griensven M, et al. Pulmonary microbial infection in mice: comparison of different application methods and correlation of bacterial numbers and histopathology. Exp Toxicol Pathol. 2002; 54(2):127-33.

15. Khavkin T, Tabibzadeh SS. Histologic, immunofluorescence, and electron microscopic study of infectious process in mouse lung after intranasal challenge with Coxiella burnetii. Infect Immun. 1988; 56(7):1792-9.

16. Kishimoto RA, Burger GT. Appearance of cellular and humoral immunity in guinea pigs after infection with Coxiella burnetii administered in small-particle aerosols. Infect Immun. 1977; 16(2):518-21.

17. Russell-Lodrigue KE, Zhang GQ, McMurray DN, Samuel JE. Clinical and pathologic changes in a guinea pig aerosol challenge model of acute Q fever. Infect Immun. 2006; 74(11):6085-91.

18. Sidwell RW, Thorpe BD, Gebhardt LP. Studies of Latent Q Fever Infections. li. Effects of Multiple Cortisone Injections. Am J Hyg. 1964; 79:320-7.

19. Baumgartner W, Dettinger $\mathrm{H}$, Schmeer N. Spread and distribution of Coxiella burnetii in C57BL/6J $(\mathrm{H}-$ $2 b)$ and Balb/cJ (H-2d) mice after intraperitoneal infection. J Comp Pathol. 1993; 108(2):165-84.

20. Meghari S, Bechah Y, Capo C, Lepidi H, Raoult D, Murray PJ, et al. Persistent Coxiella burnetii infection in mice overexpressing IL-10: an efficient model for chronic Q fever pathogenesis. PLoS Pathog. 2008; 4(2):e23.

21. Elliott A, Peng Y, Zhang G. Coxiella burnetii interaction with neutrophils and macrophages in vitro and in SCID mice following aerosol infection. Infect Immun. 2013; 81(12):4604-14.

22. Marrie TJ, Stein A, Janigan D, Raoult D. Route of infection determines the clinical manifestations of acute Q fever. J Infect Dis. 1996; 173(2):484-7.

23. Waag DM, Byrne WR, Estep J, Gibbs P, Pitt ML, Banfield CM. Evaluation of cynomolgus (Macaca fascicularis) and rhesus (Macaca mulatta) monkeys as experimental models of acute $Q$ fever after aerosol exposure to phase-I Coxiella burnetii. Lab Anim Sci. 1999; 49(6):634-8. 
24. Driscoll KE, Costa DL, Hatch G, Henderson R, Oberdorster G, Salem H, et al. Intratracheal instillation as an exposure technique for the evaluation of respiratory tract toxicity: uses and limitations. Toxicol Sci. 2000; 55(1):24-35.

25. Omsland A, Cockrell DC, Fischer ER, Heinzen RA. Sustained axenic metabolic activity by the obligate intracellular bacterium Coxiella burnetii. J Bacteriol. 2008; 190(9):3203-12.

26. Bivas-Benita $M$, Zwier R, Junginger HE, Borchard G. Non-invasive pulmonary aerosol delivery in mice by the endotracheal route. Eur J Pharm Biopharm. 2005; 61(3):214-8.

27. Dupont HT, Thirion X, Raoult D. Q fever serology: cutoff determination for microimmunofluorescence. Clin Diagn Lab Immunol. 1994; 1(2):189-96.

28. Bewley KR. Animal models of Q fever (Coxiella burnetii). Comp Med. 2013; 63(6):469-76.

29. Norville IH, Hartley MG, Martinez E, Cantet F, Bonazzi M, Atkins TP. Galleria mellonella as an alternative model of Coxiella burnetii infection. Microbiology. 2014; 160(Pt 6):1175-81.

30. Norville IH, Hatch GJ, Bewley KR, Atkinson DJ, Hamblin KA, Blanchard JD, et al. Efficacy of liposomeencapsulated ciprofloxacin in a murine model of Q fever. Antimicrob Agents Chemother. 2014; 58(9):5510-8.

31. La Scola B, Lepidi $H$, Raoult D. Pathologic changes during acute $Q$ fever: influence of the route of infection and inoculum size in infected guinea pigs. Infect Immun. 1997; 65(6):2443-7.

32. Wielders CC, Wuister AM, de Visser VL, de Jager-Leclercq MG, Groot CA, Dijkstra F, et al. Characteristics of hospitalized acute $Q$ fever patients during a large epidemic, The Netherlands. PLoS One. 2014; 9(3):e91764.

33. Lee M, Jang JJ, Kim YS, Lee SO, Choi SH, Kim SH, et al. Clinicopathologic features of q Fever patients with acute hepatitis. Korean J Pathol. 2012; 46(1):10-4.

34. Eldin C, Melenotte C, Mediannikov O, Ghigo E, Million M, Edouard S, et al. From Q Fever to Coxiella burnetii Infection: a Paradigm Change. Clin Microbiol Rev. 2017; 30(1):115-90.

\section{Figures}



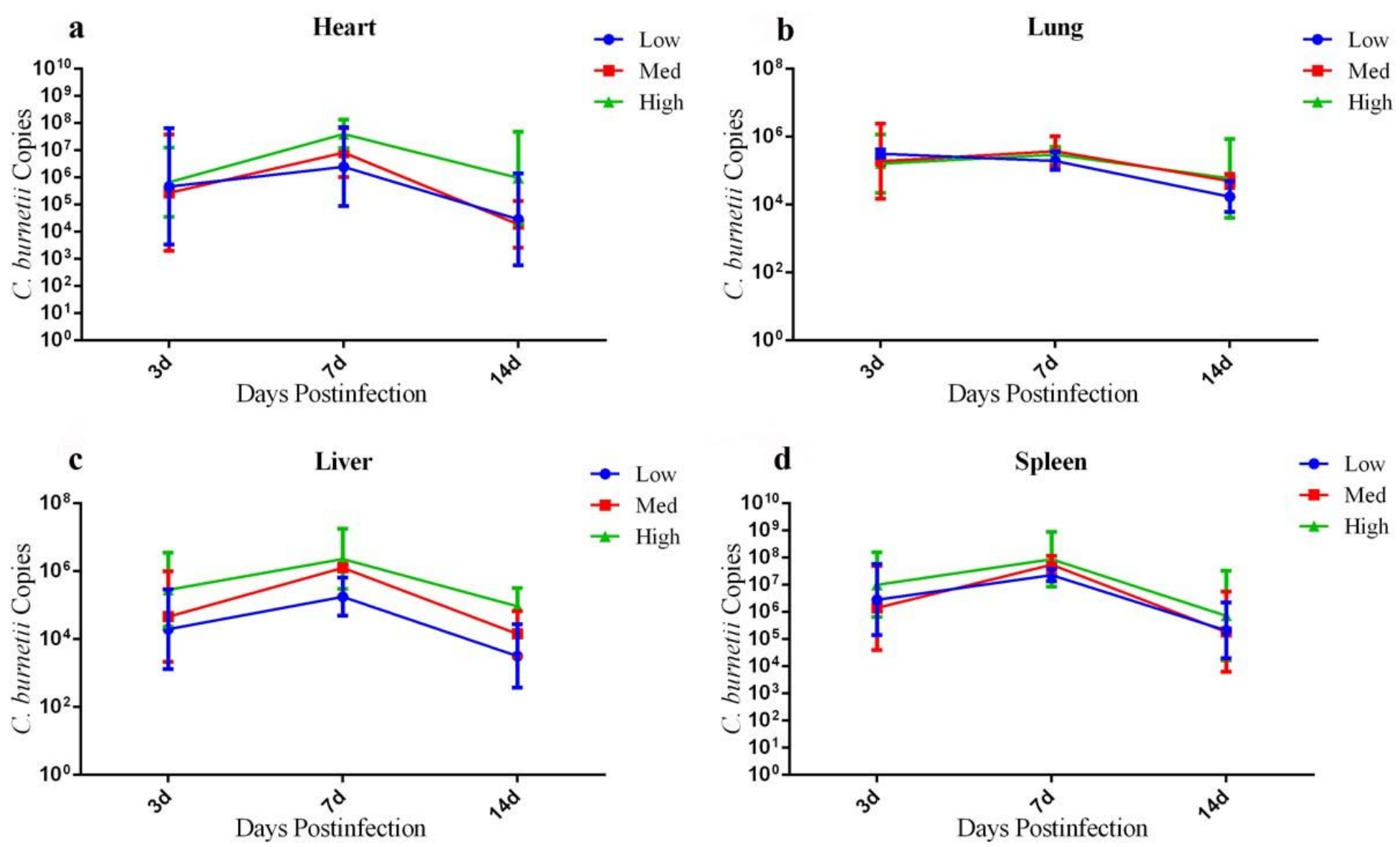

Figure 1

C. burnetii loads in organs of mice infected with $\mathrm{C}$. burnetii by IT route. BALB/c Mice received an inoculum of 1×104 (Low), 1×106 (Med), and 1×108 (High) C. burnetii Xinqiao strain, respectively, via IT route (IT). Mice were killed at day 3,7 , and 14 pi, respectively. 

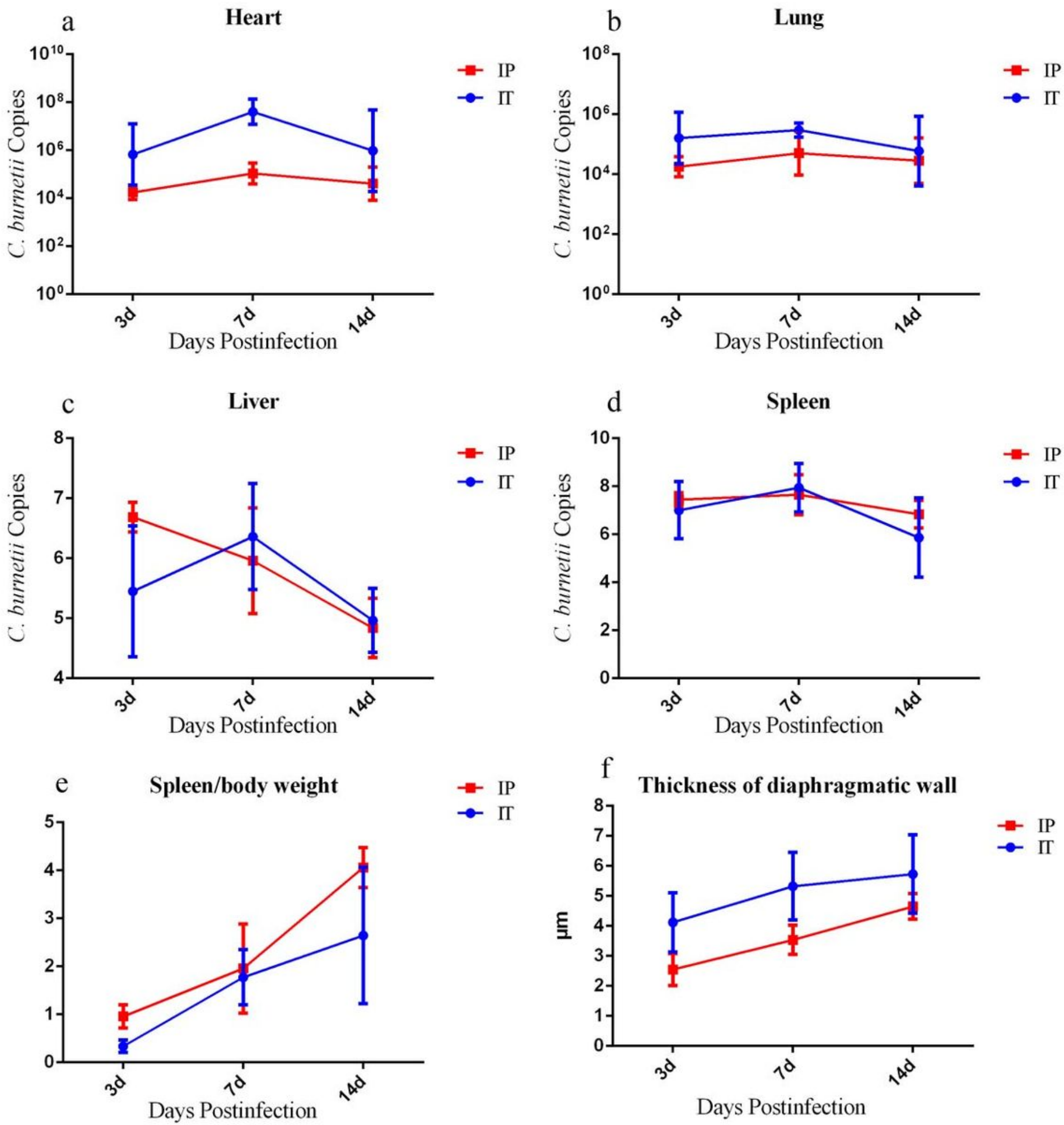

Figure 2

C. burnetii loads in organs, spleen/body weight and thickness of diaphragmatic wall of mice infected with $\mathrm{C}$. burnetii by IT route or IP route. BALB/c Mice received an inoculum of $1 \times 108 \mathrm{C}$. burnetii Xinqiao strain via IT route $(\mathrm{IT})$. Mice received $1 \times 108 \mathrm{C}$. burnetii Xinqiao strain via IP route (IP) as the control. Mice were killed at day 3,7 , and 14 pi, respectively. a: C. burnetii loads in hearts; b: C. burnetii loads in lungs; c: 
C. burnetii loads in livers; $d$ : C. burnetii loads in spleens; e: spleen/body weight; f: thickness of diaphragmatic wall.

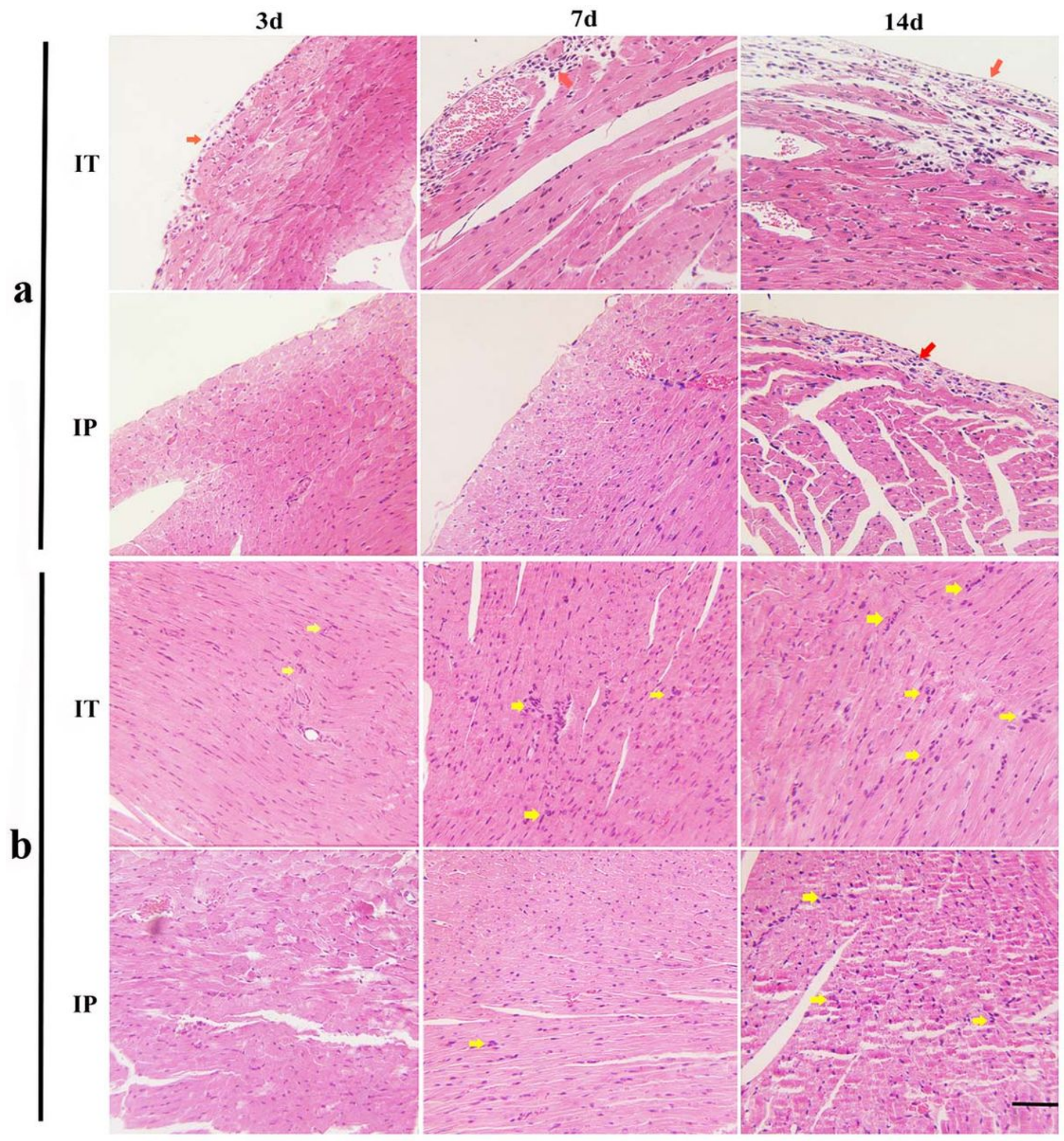

\section{Figure 3}

Histopathological analysis in hearts of mice infected with C. burnetii by IT route or IP route. BALB/c mice received an inoculum of $1 \times 108 \mathrm{C}$. burnetii Xinqiao strain via IT route or IP route. a: histopathological lesions in epicardium; b: histopathological lesions in myocardium. A large amount of inflammatory cells 
in epicardium (shown with red arrows) and the infiltration of inflammatory cells in myocardium (shown with yellow arrows) were observed of mice infected via IT route at day 3 pi. The infiltration of inflammatory cells in myocardium (shown with yellow arrows) were observed of mice infected via IP route at day $7 \mathrm{pi}$ and a small amount of inflammatory cells in epicardium were noted at day $14 \mathrm{pi}$. Original magnification, $200 \times$. Scale bars, $100 \mu \mathrm{m}$.

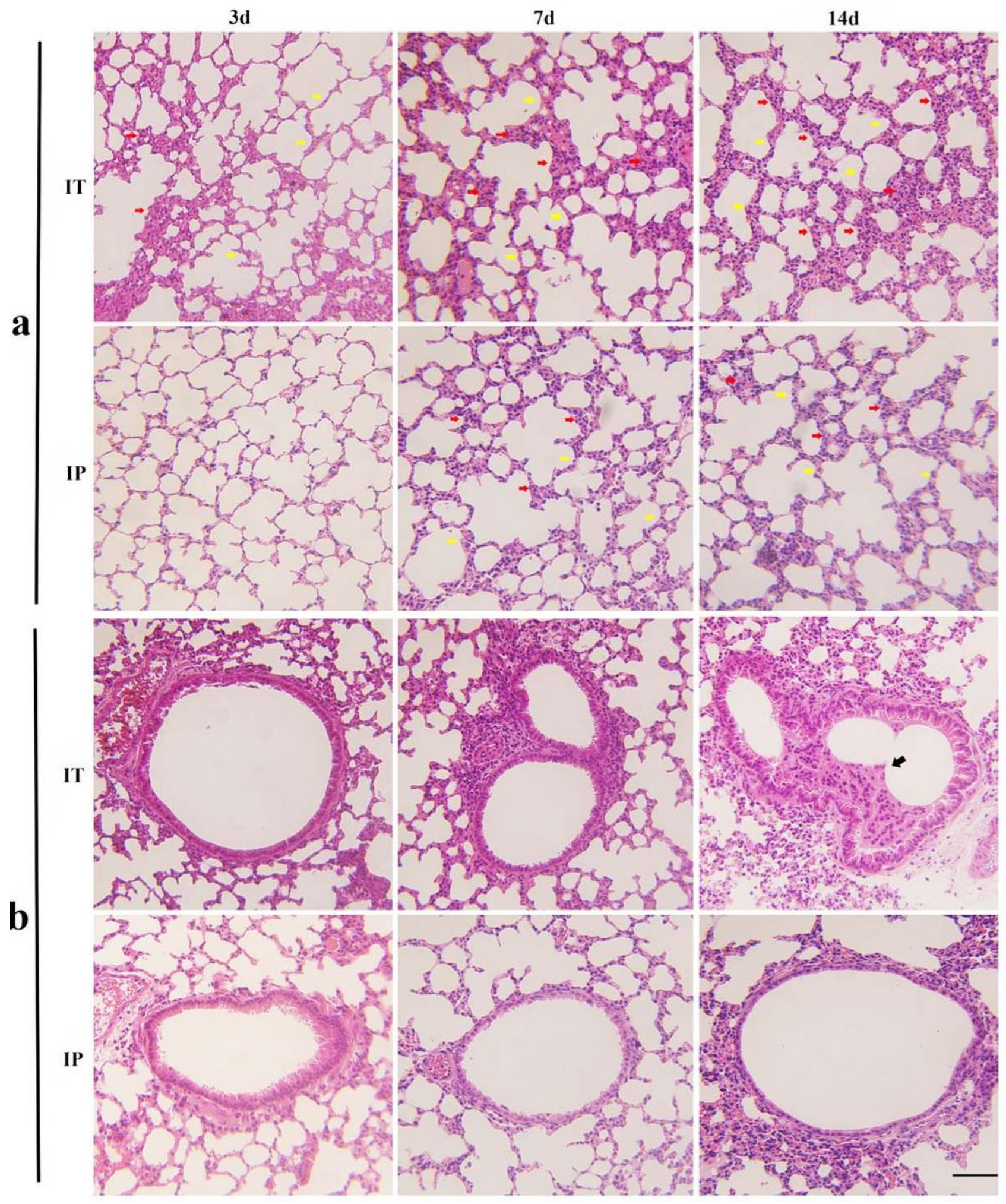

Figure 4 
Histopathological analysis in lungs of mice infected with $C$. burnetii by IT route or IP route. BALB/c mice received an inoculum of $1 \times 108 \mathrm{C}$. burnetii Xinqiao strain via IT route or IP route. a: histopathological lesions in lungs ; $b$ : histopathological lesions in tracheas. The infiltration of inflammation cells (shown with red arrows) and the increased thickness of diaphragmatic walls (shown with yellow arrows) were observed in the lungs of mice infected via IT route at day 3 pi and in the lungs of mice infected via IP route at day $7 \mathrm{pi}$. Inflammation cells and epithelial cells (shown with black arrows) appeared only in tracheas of mice infected via IT route at day 14 pi. Original magnification, $200 \times$. Scale bars, $100 \mu \mathrm{m}$.

3d
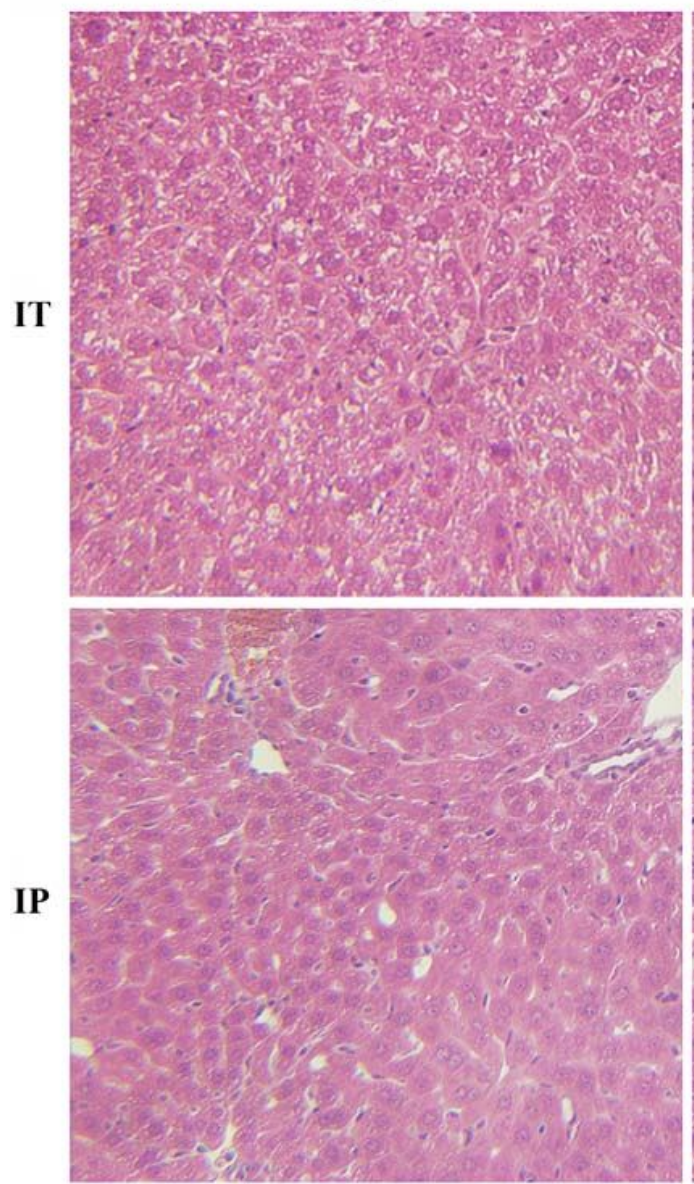

$7 d$
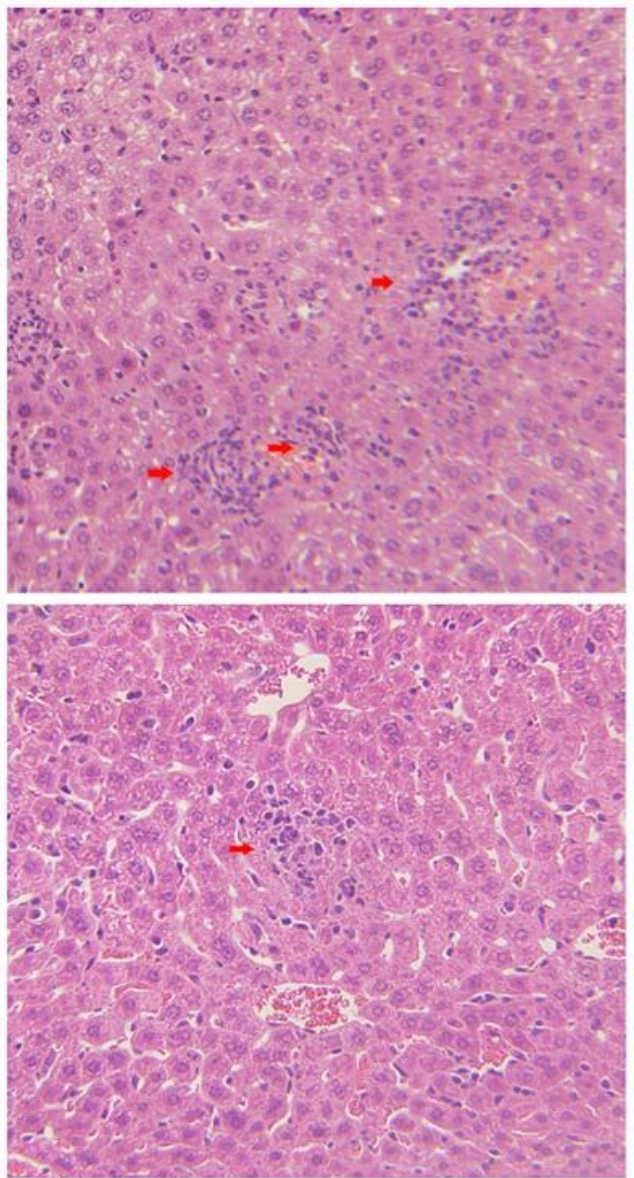

14d

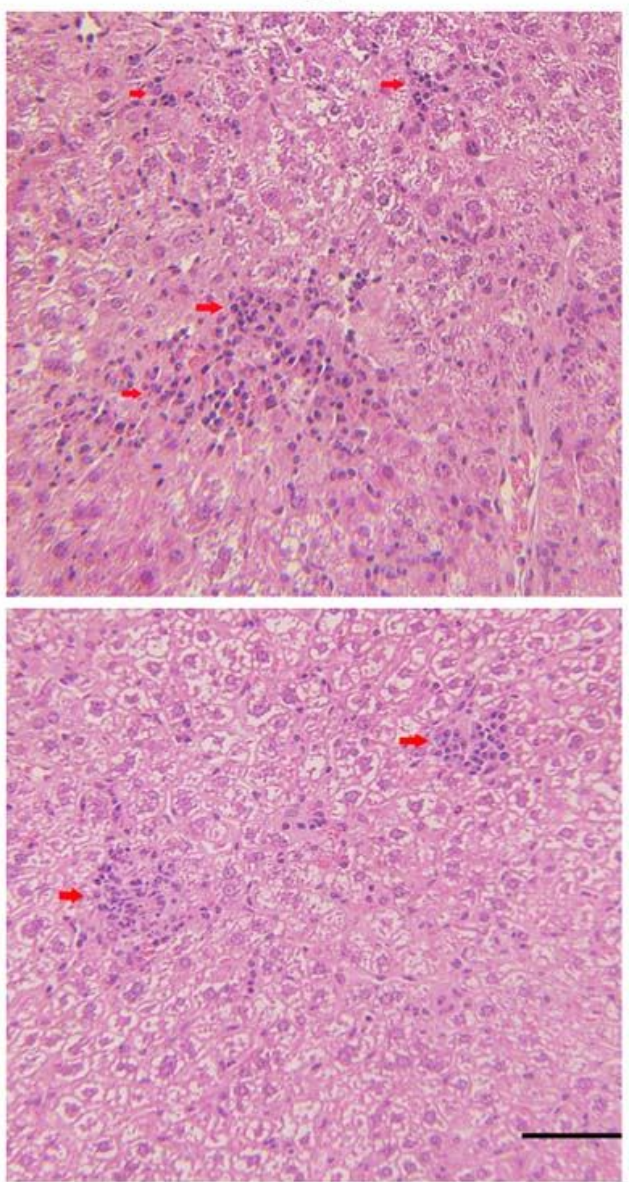

\section{Figure 5}

Histopathological analysis in livers of mice infected with $\mathrm{C}$. burnetii by IT route or IP route. BALB/c mice received an inoculum of $1 \times 108 \mathrm{C}$. burnetii Xinqiao strain via IT route or IP route. The infiltration of inflammatory cells (shown with red arrows) were observed in the livers of mice infected with $\mathrm{C}$. burnetii via IT or IP route at day $7 \mathrm{pi}$. Original magnification, $200 \times$. Scale bars, $100 \mu \mathrm{m}$. 
$14 d$

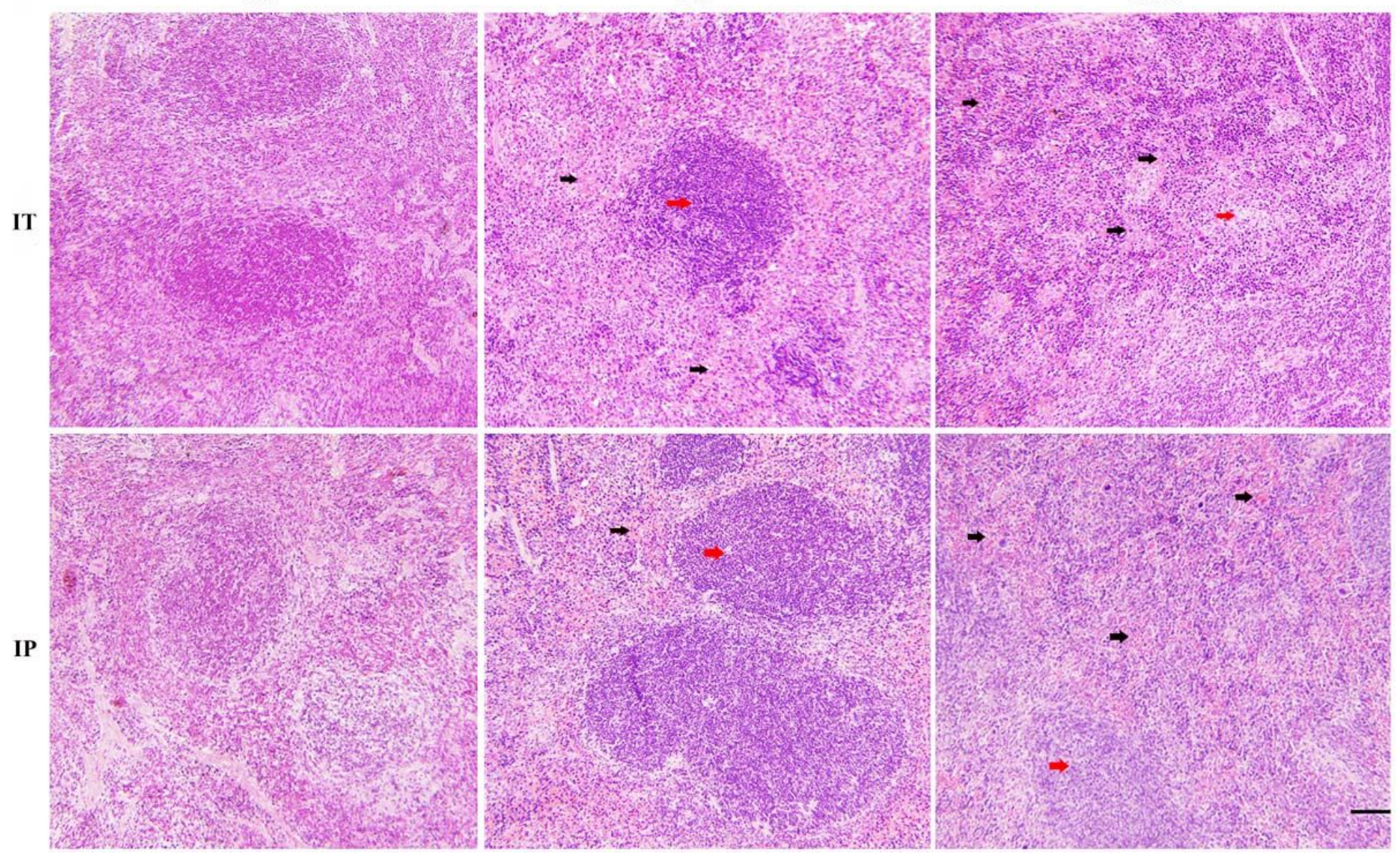

\section{Figure 6}

Histopathological analysis in spleens of mice infected with $\mathrm{C}$. burnetii by IT route or IP route. BALB/C mice received an inoculum of $1 \times 108 \mathrm{C}$. burnetii Xinqiao strain via IT route or IP route. Red pulp (shown with black arrows) widened and white pulp (shown with red arrows) atrophied, and splenic sinusoid extended with hyperemia, and the boundary became blurred in the spleens of mice infected with $\mathrm{C}$. burnetii via IT or IP route at day 7 pi. Original magnification, $100 \times$. Scale bars, $100 \mu \mathrm{m}$.
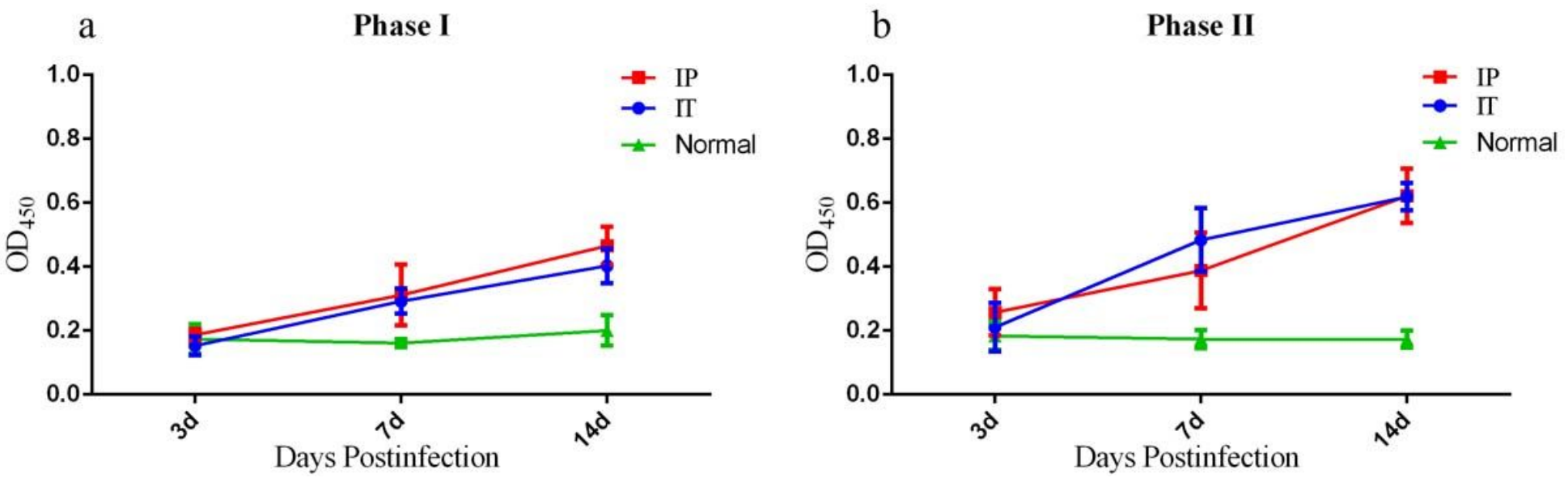

Figure 7 
Antibody response in sera of mice infected with $\mathrm{C}$. burnetii by IT route or IP route. BALB/c Mice received an inoculum of $1 \times 108 \mathrm{C}$. burnetii Xinqiao strain via IT route and IP route, respectively. Normal mice without infection were regarded as a negative control. Mice were killed at day 3, 7, and 14 pi, respectively. C. burnetii-specific IgG responses in sera were measured. OD450, optical density at $450 \mathrm{~nm}$. a: the antibody levels to phase I antigen; $b$ : the antibody levels to phase II antigen.

\section{Supplementary Files}

This is a list of supplementary files associated with this preprint. Click to download.

- NC3RsARRIVEGuidelinesChecklist.docx 